\title{
Generalized stochastic Lagrangian paths for the Navier-Stokes equation
}

\author{
Marc Arnaudon $^{a} \quad$ Ana Bela Cruzeiro $^{b}$ and Shizan Fang ${ }^{c}$ \\ ${ }^{a}$ Institut de Maths. de Bordeaux, Université de Bordeaux I, 33405 Talence Cedex, France \\ ${ }^{b}$ GFMUL and Dep. de Mat. Instituto Superior Técnico, Av. Rovisco Pais, 1049-001 Lisboa, Portugal \\ ${ }^{c}$ Institut de Maths. de Bourgogne, Université de Bourgogne, 21078 Dijon Cedex, France
}

February 18th, 2016

\begin{abstract}
In the note added in proof of the seminal paper [Groups of diffeomorphisms and the motion of an incompressible fluid, Ann. of Math. 92 (1970), 102-163], Ebin and Marsden introduced the so-called correct Laplacian for the Navier-Stokes equation on a compact Riemannian manifold. In spirit of Brenier's generalized flows for the Euler equation, we introduce a class of semimartingales on a compact Riemannian manifold. We prove that these semimartingales are critical points to the corresponding kinetic energy if and only if its drift term solves weakly the Navier-Stokes equation defined with Ebin-Marsden's Laplacian. We also show that for the case of torus, classical solutions of the Navier-Stokes equation realize the minimum of the kinetic energy in a suitable class.
\end{abstract}

\section{Introduction}

Euler equations describe the velocity of incompressible non-viscous fluids. Considering these equations on a bounded domain $U$ of $\mathbb{R}^{d}$, or on a compact Riemannian manifold $M$ without boundary, they read

$$
\frac{d}{d t} u_{t}+\left(u_{t} \cdot \nabla\right) u_{t}=-\nabla p, \quad \operatorname{div}\left(u_{t}\right)=0
$$

Lagrange's point of view consists in describing the position of the particles: for a solution $u$ to (1.1), it concerns solutions of the ordinary differential equation (ODE)

$$
\frac{d}{d t} g_{t}(x)=u_{t}\left(g_{t}(x)\right), \quad g_{0}(x)=x .
$$

When $(t, x) \rightarrow u_{t}(x)$ is smooth, the ODE (1.2) defines a flow of $C^{\infty}$-diffeomorphisms $g_{t}$. From the position values, we get the velocity by

$$
u_{t}(x)=\left(\frac{d}{d t} g_{t}\right)\left(g_{t}^{-1}(x)\right)
$$

In this case, the two points of view are equivalent. Throughout the whole paper we shall consider the interval of time $[0, T]$. Equation (1.2) defines a continuous map

$$
\text { g. : }[0, T] \rightarrow \operatorname{Diff}(M)
$$


from $[0, T]$ to the group of diffeomorphisms of $M$.

In a famous work [6], V.I. Arnold gave a geometric interpretation to the incompressible Euler equation, saying that $u$ is a solution to (1.1) if and only if $t \rightarrow g_{t}$ is a geodesic on the submanifold of $\operatorname{Diff}(M)$ keeping the volume measure invariant, equipped with the $L^{2}$ metric. Equivalently, g. minimizes the action

$$
S[\varphi]=\frac{1}{2} \int_{0}^{T} \int_{M}\left|\frac{d}{d t} \varphi_{t}(x)\right|_{T_{x} M}^{2} d x d t
$$

on $C([0, T]$, Diff $(M))$, where $d x$ denotes the normalized Lebesgue measure on $U$ or the normalized Riemannian volume on $M$ (see also [14]).

In [7, Y. Brenier gave a probabilistic interpretation to (1.1), by looking for probability measures $\eta$ on the path space $C([0, T], M)$, which minimize the kinetic energy

$$
S[\eta]=\frac{1}{2} \int_{C([0, T], M)}\left[\int_{0}^{T}|\dot{\gamma}(t)|_{T_{\gamma(t)} M}^{2} d t\right] d \eta(\gamma),
$$

with constraints $\left(e_{t}\right)_{*} \eta=d x$, where $e_{t}: \gamma \rightarrow \gamma(t)$ denotes the evaluation map. Let

$$
X(\gamma, t)=\gamma(t)
$$

Then under $\eta,\{X(\cdot, t) ; t \geq 0\}$ is a $M$-valued stochastic process. Moreover, in [7] as well as in [8], Brenier proved that such a probability measure $\eta$ gives rise to a weak solution of the Euler equation in the sense of Di Perna and Majda [13. More precisely, define a probability measure $\mu$ on $[0, T] \times T M$ by

$$
\begin{aligned}
& \int_{[0, T] \times T M} f(t, x, v) \mu(d t, d x, d v) \\
& =\frac{1}{T} \int_{0}^{T} \int_{C([0, T], M)} f\left(t, \gamma(t), \gamma^{\prime}(t)\right) d \eta(\gamma) d t .
\end{aligned}
$$

Then $\mu$ solves the Euler equation in generalized sense:

$$
\int\left[v \cdot w(x) \alpha^{\prime}(t)+v \cdot(\nabla w(x) \cdot v) \alpha(t)\right] \mu(d t, d x, d v)=0
$$

for any $\alpha \in C^{\infty}(] 0, T[)$ and any smooth vector field $w \operatorname{such}$ that $\operatorname{div}(w)=0$. We also refer to [1] in which the authors used the theory of mass transportation.

In this work, we will deal with Navier-Stokes equations on a compact Riemannian manifold $M$. There are two natural ways to define the "Laplace" operator on vector fields. The first way is to use the de Rham-Hodge Laplace operator $\square$ on differential 1-forms, that is $\square=d d^{*}+d^{*} d$. As usual, for a vector field $A$, we denote by $A^{\sharp}$ the associated differential 1-form; for a differential 1-form $\omega$, we denote by $\omega^{b}$ the corresponding vector field. Then we define

$$
\square A=\left(\square A^{\sharp}\right)^{b} .
$$

The Weitzenböck formula states that

$$
-\square A=\Delta A-\operatorname{Ric}(A)
$$


where $\Delta A=\operatorname{Trace}\left(\nabla^{2} A\right)$ and Ric is the Ricci tensor. Another natural way, following [14, is to use the deformation tensor. More precisely, let $A$ be a vector field on $M$, the deformation tensor Def $A$ is a symmetric tensor of type $(0,2)$ such that

$$
(\operatorname{Def} A)(X, Y)=\frac{1}{2}\left(\left\langle\nabla_{X} A, Y\right\rangle+\left\langle\nabla_{Y} A, X\right\rangle\right) .
$$

Then Def : $T M \rightarrow S^{2} T^{*} M$ which sends a vector field to a symmetric tensor of type (0,2). Let Def* $: S^{2} T^{*} M \rightarrow T M$ be the adjoint operator. According to [25], as well as to [27], we define

$$
\hat{\square}=2 \operatorname{Def}^{*} \text { Def. }
$$

Then on vector fields of divergence free $A$, it holds true (see [25, 26])

$$
-\hat{\square} A=\Delta A+\operatorname{Ric}(A) \text {. }
$$

Comparing (1.8) to (1.5), the sign of Ric is opposite.

In this work, we will consider the following Navier-Stokes equation on $M$

$$
\frac{d}{d t} u_{t}+\nabla_{u_{t}} u_{t}+\nu \hat{\square} u_{t}=-\nabla p, \operatorname{div}\left(u_{t}\right)=0,
$$

where $\nu>0$ is the viscosity coefficient. Since $\operatorname{div}\left(u_{t}\right)=0$, we have $\int_{M}\left\langle\nabla_{u_{t}} u_{t}, u_{t}\right\rangle d x=0$. Using the relation $\hat{\square}=\square-2$ Ric and equation (1.9), we get

$$
\frac{1}{2} \frac{d}{d t} \int_{M}\left|u_{t}\right|^{2} d t+\nu \int_{M}\left(\left|d u_{t}^{\sharp}\right|^{2}+\left|d^{*} u_{t}^{\sharp}\right|^{2}\right) d x-2 \nu \int_{M}\left\langle\operatorname{Ric} u_{t}, u_{t}\right\rangle d x=0 .
$$

When Ric is negative, the above relation yields the existence of Leray's weak solution (see for instance [26]). For the general case, the existence of Leray's weak solution to (1.9) was proved in [27] (Theorem 4.6, p.498 and p.504).

In contrast to Euler equations, there is no geometrical interpretation for Navier-Stokes equations. The purpose of this work is to develop a probabilistic interpretation to equation (1.9). Note that in this context, it is suitable to consider that the underlying Lagrangian trajectories are semimartingales $\xi_{t}$ on the manifold $M$. Comparing to Brenier's generalized flows for Euler equations, the paths $t \rightarrow \xi_{t}$ are never of finite energy in the sense of (1.3). Instead, we shall consider the mean kinetic energy (see definition (2.13) below). This functional first appeared in stochastic optimal control [18 as well as in connection with quantum mechanics [29]; we mention also [19] for the relation of (stochastic) kinetic energy and entropy as well as [28], for its appereance in the study of the Navier-Stokes equation.

Roughly speaking, the main result of this paper (see Theorem 2.10 below) says that the semi-martingale $\xi_{t}$ in a suitable class is a critical point to the stochastic kinetic energy (2.13) if and only if its drift term $u_{t}$ solves Navier-Stokes equation (1.9) in the sense of Di-Perna and Majda.

In the recent years the functional (2.13) has been used with success in various contexts (see for example [2, 3, 4, 5, 10, 11, 12, 15, 20, 23). In comparison with [2, 3, 4, 5, we do not require, in the present work, that martingales have the flow property.

The organisation of the paper is as follows. In section 2, we shall introduce and study the class of $\nu$-Brownian incompressible semimartingales. We prove that such a semimartingale is a critical point of the corresponding kenetic energy [12] if and only if it solves the Navier-Stokes equation in the sense of DiPerna-Majda [7, 8]. We also prove the existence 
of a minimum under certain conditions. In section 3, we shall show, in the case of a torus $\mathbb{T}^{d}$, that a classical solution to Navier-Stokes equation gives rise to a $\nu$-Brownian incompressible martingale which realizes the minimum of the kinetic energy in a convenient class.

\section{Generalized stochastic paths for the Navier-Stokes equa- tion}

In this section, $M$ will denote a connected compact Riemannian manifold without boundary. Let $(\Omega, \mathcal{F}, P)$ be a probability space equipped with a filtration $\left\{\mathcal{F}_{t} ; t \geq 0\right\}$ satisfying the usual conditions.

A $M$-valued stochastic process $\xi_{t}$ defined on $(\Omega, \mathcal{F}, P)$ is said to be a semimartingale on $M$ if for any $f \in C^{2}(M), f\left(\xi_{t}\right)$ is a real valued semimartingale. This notion is independent of the chosen connection on $M$; however, the corresponding local characteristics are dependent of the choice of connection. For a semimartingale $\left(\xi_{t}\right)$ starting from a point $x \in M$ and given a connection $\nabla$, the stochastic parallel translation $/ / t$ along $\xi$. can be defined and

$$
\zeta_{t}=\int_{0}^{t} / /_{s}^{-1} \circ d \xi_{s}
$$

is a $T_{x} M$-valued semimartingale. Then there exist processes $\left(\xi^{0}(s), H_{1}(s), \ldots, H_{m}(s)\right)$ which are adapted to $\mathcal{F}_{t}$ such that

$$
\xi^{0}(s), H_{1}(s), \ldots, H_{m}(s) \in T_{\xi_{s}} M
$$

and $\zeta_{t}$ admits Itô form

$$
\zeta_{t}=\int_{0}^{t} / /_{s}^{-1} \xi^{0}(s) d s+\sum_{i=1}^{m} \int_{0}^{t} / /_{s}^{-1} H_{i}(s) d w_{s}^{i}
$$

where $w_{t}=\left(w_{t}^{1}, \cdots, w_{t}^{m}\right)$ is a standard Brownian motion on $\mathbb{R}^{m}$ (see for example [9]). For example, if the semimartingale $\xi_{t}$ comes from a stochastic differential equation (SDE) on $M:$

$$
d \xi_{t}=X_{0}\left(t, \xi_{t}\right) d t+\sum_{i=1}^{m} X_{i}\left(t, \xi_{t}\right) \circ d w_{t}^{i}, \quad \xi_{0}=x,
$$

then

$$
\xi^{0}(t)=X_{0}\left(t, \xi_{t}\right)+\frac{1}{2} \sum_{i=1}^{m}\left(\nabla_{X_{i}} X_{i}\right)\left(t, \xi_{t}\right) .
$$

For simplicity, in what follows, we only consider the Levi-Civita connection $\nabla$ on $M$. As in [3], [12, we consider the operator

$$
D_{t} \xi=/ / t \lim _{\varepsilon \rightarrow 0} \mathbb{E}\left(\frac{\zeta_{t+\varepsilon}-\zeta_{t}}{\varepsilon} \mid \mathcal{F}_{t}\right),
$$

which is well-defined and equals $\xi^{0}(t)$. For a semimartingale $\xi_{t}$ given by (2.1), the Itô formula has the following form (see [9], p. 409) 


$$
\begin{aligned}
f\left(\xi_{t}\right)=f\left(\xi_{0}\right) & +\int_{0}^{t}\left(\left\langle\nabla f\left(\xi_{s}\right), \xi^{0}(s)\right\rangle+\frac{1}{2} \sum_{i=1}^{m}\left\langle\nabla_{H_{i}(s)}(\nabla f)\left(\xi_{s}\right), H_{i}(s)\right\rangle\right) d s \\
& +\sum_{i=1}^{m} \int_{0}^{t}\left\langle\nabla f\left(\xi_{s}\right), H_{i}(s)\right\rangle d w_{s}^{i} .
\end{aligned}
$$

Let $\left\{g_{t}(x, \omega) ; t \geq 0, x \in M, \omega \in \Omega\right\}$ be a family of continuous semimartingales with values in $M$. Let $\mathbb{P}^{g}$ denote the law of $g$ in the continuous path space $C([0, T], M)$, that is, for every cylindrical functional $F$,

$$
\int_{C([0, T], M)} F\left(\gamma\left(t_{1}\right), \ldots, \gamma\left(t_{n}\right)\right) d \mathbb{P}^{g}(\gamma)=\int_{M}\left[\int_{C([0, T], M)} F\left(g_{t_{1}}(x), \ldots, g_{t_{n}}(x)\right) d \mathbb{P}_{x}^{g}\right] d x
$$

where $\mathbb{P}^{g}=\mathbb{P}_{x}^{g} \otimes d x$ and under $\mathbb{P}_{x}^{g}$, the semimartingale $g_{t}$ starts from $x$.

We shall say that the semimartingale $g_{t}$ is incompressible if, for each $t>0$,

$$
\mathbb{E}_{\mathbb{P} g}\left[f\left(g_{t}\right)\right]=\int_{M} f(x) d x, \quad \text { for all } f \in C(M)
$$

the expectation being taken with respect to the law $\mathbb{P}^{g}$ of $g$.

Let $\nu>0$; we shall say that $g_{t}$ is a $\nu$-Brownian semimartingale if, under $\mathbb{P}^{g}$, there exists a time-dependent adapted random vector field $u_{t}$ over $g_{t}$ such that

$$
M_{t}^{f}=f\left(g_{t}\right)-f\left(g_{0}\right)-\int_{0}^{t}\left(\nu \Delta f\left(g_{s}\right)+\left\langle u_{s}, \nabla f\left(g_{s}\right)\right\rangle\right) d s,
$$

is a local continuous martingale with the quadratic variation given by

$$
\left\langle M_{t}^{f_{1}}, M_{t}^{f_{2}}\right\rangle=2 \nu \int_{0}^{t}\left\langle\nabla f_{1}, \nabla f_{2}\right\rangle\left(g_{s}\right) d s .
$$

For a semimartingale $\xi_{t}$ given by (2.1), if $\left\{H_{1}(s), \ldots, H_{m}(s)\right\}$ is an orthogonal system such that for any vector $v \in T_{\xi_{s}} M, \sum_{i=1}^{m}\left\langle v, H_{i}(s)\right\rangle^{2}=2 \nu|v|^{2}$, then it is a $\nu$-Brownian semimartingale.

Example 2.1. In the flat case $\mathbb{R}^{d}$, such a semimartingale admits the following form

$$
d g_{t}(w)=\sqrt{2 \nu} d w_{t}+u_{t}(w) d t
$$

where $\left(w_{t}\right)$ is a Brownian motion on $\mathbb{R}^{d}$ and $\left\{u_{t} ; t \geq 0\right\}$ is an adapted $\mathbb{R}^{d}$-valued process such that $\int_{0}^{T}\left|u_{t}(w)\right|^{2} d t<+\infty$ almost surely.

Example 2.2. For the general case of a compact Riemannian manifold $M$, we consider the bundle of orthonormal frames $O(M)$. Let $\left(V_{t}\right)_{t \in[0, T]}$ be a family of $C^{1}$ vector fields such that the dependence $t \rightarrow V_{t}$ is $C^{1}$. Denote by $\tilde{V}_{t}$ the horizontal lift of $V_{t}$ to $O(M)$. Let $\operatorname{div}\left(V_{t}\right)$ and $\operatorname{div}\left(\tilde{V}_{t}\right)$ be respectively the divergence operators on $M$ and on $O(M)$; they are linked by (see [16], p. 595)

$$
\operatorname{div}\left(\tilde{V}_{t}\right)=\operatorname{div}\left(V_{t}\right) \circ \pi
$$


where $\pi: O(M) \rightarrow M$ is the canonical projection. It follows that if $\operatorname{div}\left(V_{t}\right)=0$, then $\operatorname{div}\left(\tilde{V}_{t}\right)=0$. Consider the horizontal diffusion $r_{t}$ on $O(M)$ defined by the SDE

$$
d r_{t}=\sqrt{2 \nu} \sum_{i=1}^{d} H_{i}\left(r_{t}\right) \circ d w_{t}^{i}+\tilde{V}_{t}\left(r_{t}\right) d t, \quad r_{0} \in O(M)
$$

where $\left\{H_{1}, \cdots, H_{d}\right\}$ are the canonical horizontal vector fields on $O(M)$. Let $d r$ be the Liouville measure on $O(M)$, then the stochastic flow $r_{0} \rightarrow r_{t}\left(r_{0}\right)$ leaves $d r$ invariant. Set

$$
\xi(t, x)=\pi\left(r_{t}\left(r_{0}\right)\right), \quad r_{0} \in \pi^{-1}(x) .
$$

For any continuous function $f$ on $M$,

$$
\int_{M} \mathbb{E}\left(f(\xi(t, x)) d x=\int_{M} f(x) d x .\right.
$$

Then $\xi$ is an incompressible $\nu$-Brownian diffusion, with $D_{t} \xi(x)=V_{t}(\xi(t, x))$.

Remark 2.3. Let $P_{t}$ be the semigroup associated to $\frac{1}{2} \Delta_{M}+V_{t}$ with $\operatorname{div}\left(V_{t}\right)=0$; then for any $f \in C^{2}(M)$,

$$
\frac{d}{d t} \int_{M} P_{t} f(x) d x=\int_{M}\left(\frac{1}{2} \Delta_{M} P_{t} f+V_{t} P_{t} f\right) d x=0 .
$$

It follows that for any continuous function $f: M \rightarrow \mathbb{R}$,

$$
\int_{M} P_{t} f(x) d x=\int_{M} f(x) d x .
$$

Therefore any SDE on $M$ defining a Brownian motion with drift $V$ gives rise to an incompressible $\nu$-Brownian diffusion $\xi$ with $D_{t} \xi(x)=V_{t}(\xi(t, x))$.

Example 2.4. Let $\mathbb{Z}^{2}$ be the set of two dimensional lattice points and define $\mathbb{Z}_{0}^{2}=$ $\mathbb{Z}^{2} \backslash\left\{(0,0)^{*}\right\}$. For $k \in \mathbb{Z}_{0}^{2}$, we consider the vector $k^{\perp}=\left(k_{2},-k_{1}\right)^{*}$ and the vector fields

$$
A_{k}(\theta)=\sqrt{\frac{\nu}{\nu_{0}}} \frac{\cos (k \cdot \theta)}{|k|^{\beta}} k^{\perp}, \quad B_{k}(\theta)=\sqrt{\frac{\nu}{\nu_{0}}} \frac{\sin (k \cdot \theta)}{|k|^{\beta}} k^{\perp}, \quad \theta \in \mathbb{T}^{2},
$$

where $\beta>1$ is some constant.

Let $\tilde{\mathbb{Z}}_{0}^{2}$ the subset of $\mathbb{Z}_{0}^{2}$ where we identify vectors $k, k^{\prime}$ such that $k+k^{\prime}=0$ and let

$$
\nu_{0}=\sum_{k \in \tilde{\mathbb{Z}}_{0}^{2}} \frac{1}{2|k|^{2 \beta}} .
$$

The family $\left\{A_{k}, B_{k}: k \in \mathbb{Z}_{0}^{2}\right\}$ constitutes an orthogonal basis of the space of divergence free vector fields on $\mathbb{T}^{2}$ and satisfies

$$
\sum_{k \in \tilde{\mathbb{Z}}_{0}^{2}}\left(\left\langle A_{k}, v\right\rangle^{2}+\left\langle B_{k}, v\right\rangle^{2}\right)=\nu|v|_{T_{\theta} \mathbb{T}^{2}}^{2}, \quad v \in T_{\theta} \mathbb{T}^{2},
$$

and

$$
\sum_{k \in \tilde{\mathbb{Z}}_{0}^{2}} \nabla_{A_{k}} A_{k}=0, \sum_{k \in \tilde{\mathbb{Z}}_{0}^{2}} \nabla_{B_{k}} B_{k}=0
$$


Consider the SDE on $\mathbb{T}^{2}$,

$$
d \xi_{t}=\sum_{k \in \tilde{\mathbb{Z}}_{0}^{2}}\left(A_{k}\left(\xi_{t}\right) \circ d w_{t}^{k}+B_{k}\left(\xi_{t}\right) \circ d \tilde{w}_{t}^{k}\right)+u\left(t, \xi_{t}\right) d t, \quad \theta_{0}=\theta \in \mathbb{T}^{2}
$$

where $\left\{w_{t}^{k}, \tilde{w}_{t}^{k} ; k \in \mathbb{Z}_{0}^{2}\right\}$ are independent standard Brownian motions on $\mathbb{R}$, and $u(t, \cdot)$ is a family of divergence free vector fields in $H^{1}\left(\mathbb{T}^{2}\right)$, such that,

$$
\int_{0}^{T} \int_{\mathbb{T}^{2}}\left(|u|^{2}+|\nabla u|^{2}\right) d x d t<+\infty
$$

Then by [12, 16], for $\beta \geq 3$, the SDE (2.9) defines a stochastic flow of measurable maps which preserves the Haar measure $d x$ on $\mathbb{T}^{2}$. More precisely, for almost surely $w$, the map

$$
x \rightarrow \xi_{t}(x, w) \text { solution to (2.9) with initial condition } x
$$

leaves $d x$ invariant; this property is stronger than that of incompressibility.

In what follows, we shall denote by $\mathcal{S}$ the set of incompressible semimartingales, by $\mathcal{S}_{\nu}$ the set of incompressible $\nu$-Brownian semimartingales and by $\mathcal{D}_{\nu}$ the set of incompressible $\nu$-Brownian diffusions. Clearly we have

$$
\mathcal{D}_{\nu} \subset \mathcal{S}_{\nu} \subset \mathcal{S}
$$

Proposition 2.5. Let $g \in \mathcal{S}_{\nu}$, then for any $f \in C^{2}(M)$,

$$
\mathbb{E}_{\mathbb{P} g}\left(\left\langle\nabla f\left(g_{t}\right), u_{t}\right\rangle\right)=0 .
$$

Proof. Taking the expectation with respect to $\mathbb{P}^{g}$ in (2.5), we have

$$
\mathbb{E}_{\mathbb{P}^{g}}\left(f\left(g_{t}\right)\right)-\mathbb{E}_{\mathbb{P}^{g}}\left(f\left(g_{0}\right)\right)=\nu \int_{0}^{t} \mathbb{E}_{\mathbb{P}^{g}}\left(\Delta f\left(g_{s}\right)\right) d s+\int_{0}^{t} \mathbb{E}_{\mathbb{P}^{g}}\left(\left\langle\nabla f\left(g_{s}\right), u_{s}\right\rangle\right) d s .
$$

It follows that

$$
\nu \int_{0}^{t} \int_{M} \Delta f(x) d x d s+\int_{0}^{t} \mathbb{E}_{\mathbb{P} g}\left(\left\langle\nabla f\left(g_{s}\right), u_{s}\right\rangle\right) d s=0 .
$$

Since $\int_{M} \Delta f(x) d x=0$, we get the result.

Proposition 2.6. Let $g_{t}$ be a semimartingale on $M$ satisfying

$$
d g_{t}(x)=\sum_{i=1}^{m} A_{i}\left(g_{t}(x)\right) \circ d w_{t}^{i}+u_{t}(w, x) d x,
$$

where $A_{1}, \cdots, A_{m}$ are $C^{2}$ divergence free vector fields on $M$ and $u_{t}(w, x) \in T_{g_{t}(x)} M$ is adapted such that $\int_{M} \mathbb{E}_{x}\left(\int_{0}^{T}\left|u_{t}(w, x)\right|^{2} d t\right) d x<+\infty$; if $g$ is incompressible, then for any $f \in C^{2}(M)$

$$
\mathbb{E}_{\mathbb{P} g}\left(\left\langle\nabla f\left(g_{t}\right), u_{t}\right\rangle\right)=0 .
$$


Proof. Let $f \in C^{2}(M)$; then by Itô formula (2.3),

$$
f\left(g_{t}\right)=f\left(g_{0}\right)+M_{t}^{f}+\frac{1}{2} \sum_{i=1}^{m} \int_{0}^{t}\left(\left\langle\nabla_{A_{i}}(\nabla f), A_{i}\right\rangle+\left\langle\nabla f, \nabla_{A_{i}} A_{i}\right\rangle\right) d s+\int_{0}^{t}\left\langle\nabla f\left(g_{s}\right), u_{s}\right\rangle d s,
$$

where $M_{t}^{f}$ is the martingale part. Note that $\left\langle\nabla_{A_{i}}(\nabla f), A_{i}\right\rangle+\left\langle\nabla f, \nabla_{A_{i}} A_{i}\right\rangle=\mathcal{L}_{A_{i}} \mathcal{L}_{A_{i}} f$ where $\mathcal{L}_{A}$ denotes the Lie derivative with respect to $A$; then taking the expectation under $\mathbb{E}_{\mathbb{P}}$, we get

$$
\frac{1}{2} \sum_{i=1}^{m}\left(\int_{M} \mathcal{L}_{A_{i}} \mathcal{L}_{A_{i}} f d x\right)+\mathbb{E}_{\mathbb{P} g}\left(\left\langle\nabla f\left(g_{t}\right), u_{t}\right\rangle\right)=0 .
$$

Since for each $i, \int_{M} \mathcal{L}_{A_{i}} \mathcal{L}_{A_{i}} f d x=0$, the result follows.

In general it is not clear whether the incompressibility condition implies the relation (2.10). However, the following is true:

Proposition 2.7. Let $A_{1}, \cdots, A_{m}$ be $C^{2+\alpha}$ vector fields on $M$ and $A_{0}$ be a $C^{1+\alpha}$ vector field with some $\alpha>0$; consider

$$
d \xi_{t}(x)=\sum_{i=1}^{m} A_{i}\left(\xi_{t}(x)\right) \circ d w_{t}^{i}+A_{0}\left(\xi_{t}(x)\right) d t, \quad \xi_{0}=x .
$$

Then for almost all $w$, the map $x \rightarrow \xi_{t}(x)$ preserves the measure $d x$ if and only if $\operatorname{div}\left(A_{i}\right)=$ 0 for $i=0,1, \cdots, m$.

Proof. We give a sketch of proof (see [17] for more discussions). By [21], $; x \rightarrow \xi_{t}(x)$ is a diffeomorphism of $M$ and the push forward measure $\left(\xi_{t}^{-1}\right)_{\#}(d x)$ of $d x$ by the inverse map of $\xi_{t}$ admits the density $K_{t}$ which is given by (see [22]):

$$
K_{t}(x)=\exp \left(-\sum_{i=1}^{m} \int_{0}^{t} \operatorname{div}\left(A_{i}\right)\left(\xi_{s}(x)\right) \circ d w_{t}^{i}-\int_{0}^{t} \operatorname{div}\left(A_{0}\right)\left(\xi_{s}(x)\right) d s\right) .
$$

If $\operatorname{div}\left(A_{i}\right)=0$ for $i=0,1, \cdots, m$, it is clear that $K_{t}=1$ and $x \rightarrow \xi_{t}(x)$ preserves $d x$. Conversely, $K_{t}(x)=1$ for any $x \in M$ and $t \geq 0$ implies that,

$$
\sum_{i=1}^{m} \int_{0}^{t} \operatorname{div}\left(A_{i}\right)\left(\xi_{s}(x)\right) \circ d w_{t}^{i}+\int_{0}^{t} \operatorname{div}\left(A_{0}\right)\left(\xi_{s}(x)\right) d s=0
$$

or in Itô form:

$$
\sum_{i=1}^{m} \int_{0}^{t} \operatorname{div}\left(A_{i}\right)\left(\xi_{s}(x)\right) d w_{t}^{i}+\int_{0}^{t}\left[\frac{1}{2} \sum_{i=1^{m}} \mathcal{L}_{A_{i}} \operatorname{div}\left(A_{i}\right)+\operatorname{div}\left(A_{0}\right)\right]\left(\xi_{s}(x)\right) d s=0 .
$$

The first term of above equality is of finite quadratic variation, while the second one is of finite variation; so that for each $i=1, \cdots, m, \operatorname{div}\left(A_{i}\right)\left(\xi_{s}(x)\right)=0$ and also

$$
\left[\frac{1}{2} \sum_{i=1^{m}} \mathcal{L}_{A_{i}} \operatorname{div}\left(A_{i}\right)+\operatorname{div}\left(A_{0}\right)\right]\left(\xi_{s}(x)\right)=0 .
$$

It follows that, almost everywhere,

$$
\operatorname{div}\left(A_{i}\right)\left(\xi_{s}(x)\right)=0 \quad \text { for } \quad i=0,1, \cdots, m ;
$$

so that $\operatorname{div}\left(A_{i}\right)=0$ for $i=0,1, \cdots, m$.

According to [12, as well as [4, 20, 15, we introduce the following action functional on semimartingales. 
Definition 2.8. Let

$$
S(g)=\frac{1}{2} \mathbb{E}_{\mathbb{P}^{g}}\left(\int_{0}^{T}\left|D_{t} g\right|^{2} d t\right) .
$$

We say that $g$ has finite energy if $S(g)<\infty$.

In what follows, we shall denote more precisely $D_{t} g(x)$ for $D_{t} g$ under the law $\mathbb{P}_{x}^{g}$. Then the action defined in (2.13) can be rewritten in the following form:

$$
S(g)=\frac{1}{2} \int_{M} \mathbb{E}_{\mathbb{P}_{x}^{g}}\left(\int_{0}^{T}\left|D_{t} g(x)\right|^{2} d t\right) d x .
$$

We first recall briefly known results about the calculus of stochastic variation (see [12, 4, 10]). Let $u_{t}(x)$ be a smooth vector field on a compact manifold (or on $\mathbb{R}^{d}$ ) which, for every $t$, is of divergence zero. Consider an incompressible diffusion $g_{t}(x)$ with covariance $a$ such that $a(x, x)=2 \mu \mathfrak{g}^{-1}(x)$ where $\mathfrak{g}$ is the metric tensor and time-dependent drift $u(t, \cdot)$. It defines a flow of diffeomorphisms preserving the volume measure. We have: $D_{t} g(x)=u_{t}\left(g_{t}(x)\right)$ and

$$
S(g)=\frac{1}{2} \int_{\mathbb{T}^{d}} \mathbb{E}_{\mathbb{P}_{x}^{g}}\left(\int_{0}^{T}\left|u_{t}\left(g_{t}(x)\right)\right|^{2} d t\right) d x
$$

There are two manners to perform the perturbation.

\section{First perturbation of identity:}

Let $w$ be a smooth divergence free vector field and $\alpha \in C^{1}(] 0, T[)$. Consider, for for $\varepsilon>0$, the ODE,

$$
\frac{d \Phi_{t}^{\varepsilon}(x)}{d t}=\varepsilon \alpha^{\prime}(t) w\left(\Phi_{t}^{\varepsilon}(x)\right), \Phi_{0}(x)=x .
$$

For each $t>0, \Phi_{t}^{\varepsilon}$ is a perturbation of the identity map $i d$. By Itô's formula, for each fixed $\varepsilon>0, t \rightarrow \Phi_{t}^{\varepsilon}\left(g_{t}(x)\right)$ is a semimartingale starting from $x$. Note that $g$ and $\Phi^{\varepsilon}(g)$ are defined on the same probability space. It was proved in [12, 4] that $u$ is a weak solution to Navier-Stokes equation if and only if $g$ is a critical point of $S$. More precisely, $\frac{d}{d \varepsilon} S\left(\Phi^{\varepsilon}(g)\right)_{\left.\right|_{\varepsilon=0}}=0$ if and only if

$$
\int_{\mathbb{T}^{d}} \int_{0}^{T}\left\langle u_{t}, \alpha^{\prime}(t) w+\alpha(t) \nabla w \cdot u_{t}-\nu \alpha(t) \square w\right\rangle d t d x=0 .
$$

\section{Second perturbation of identity:}

Note that in [20], the perturbation of the identity was defined in a different way. For each fixed $t>0$, the author of [20] considered the ODE

$$
\frac{d \Psi_{s}^{t}}{d s}=\alpha(t) w\left(\Psi_{s}^{t}\right), \quad \Psi_{0}^{t}(x)=x
$$

Set $\Psi(g)_{t}^{\varepsilon}(x)=\Psi_{\varepsilon}^{t}\left(g_{t}(x)\right)$. Then $\frac{d}{d \varepsilon} S\left(\Psi(g)^{\varepsilon}\right)_{\left.\right|_{\varepsilon=0}}=0$ if and only if the equation (2.16) holds.

Now we deal with the general case of compact Riemannian manifolds. 
Definition 2.9. Let $M$ be a compact Riemannian manifold, $g$ a semimartingale on $M$ of finite energy. Define the probability measure $\mu$ on $[0, T] \times T M$ by

$$
\int_{[0, T] \times T M} f(t, x, v) \mu(d t, d x, d v)=\frac{1}{T} \mathbb{E}_{\mathbb{P} g}\left[\int_{0}^{T} f\left(t, g(t), D_{t} g\right) d t\right]
$$

where $f:[0, T] \times T M \rightarrow \mathbb{R}$ is any continuous function.

We have the following result,

Theorem 2.10. Suppose that $g \in \mathcal{S}_{\nu}$. Then $g$ is a critical point of $S$ with variations defined in (2.17) if and ony if $\mu$ is a solution to the Navier-Stokes equation in the sense of DiPerna-Majda, that is,

$$
\int_{0}^{T} \int_{T M}\left[\alpha^{\prime}(t) v \cdot w+\alpha(t) v \cdot \nabla_{v} w-\nu \alpha(t) v \cdot \hat{\square} w\right] d \mu(t, x, v)=0
$$

for all $\alpha \in C_{c}^{1}(] 0, T[)$ and all smooth vector fields $w$ such that $\operatorname{div}(w)=0$.

Proof. Let $\Psi_{\varepsilon}^{t}$ be the perturbation of identity defined in (2.17). Set $\eta_{t}^{\varepsilon}=\Psi_{\varepsilon}^{t}\left(g_{t}(x)\right)$. Then $\left\{\eta_{t}^{\varepsilon}, t \geq 0\right\}$ is a semimartingale on $M$. We denote by $\left(\xi^{0}(s), H_{1}(s), \ldots, H_{m}(s)\right)$ the local characteristics of $g_{t}(x)$. By Itô's formula (see [9], p. 408), the drift term in local characteristics of $\eta_{t}^{\varepsilon}$ is given by

$$
D_{t} \Psi_{\varepsilon}^{t}\left(g_{t}(x)\right)=\frac{\partial}{\partial t} \Psi_{\varepsilon}^{t}\left(g_{t}(x)\right)+d \Psi_{\varepsilon}^{t}\left(g_{t}(x)\right) \cdot \xi_{t}^{0}+\frac{1}{2} \sum_{i=1}^{m} \nabla\left(d \Psi_{\varepsilon}^{t}\right)\left(g_{t}(x)\right)\left(H_{i}(t), H_{i}(t)\right)
$$

where $d \Psi_{\varepsilon}^{t}\left(g_{t}(x)\right)$ denotes the differential of $\Psi_{\varepsilon}^{t}$ at $g_{t}(x)$. Let $\varphi(\varepsilon, t)=D_{t} \Psi_{\varepsilon}^{t}\left(g_{t}(x)\right) \in$ $T_{\eta_{t}^{\varepsilon}} M$; then

$$
S\left(\Psi_{\varepsilon}(g)\right)=\frac{1}{2} \mathbb{E}_{\mathbb{P}^{g}}\left(\int_{0}^{T}|\varphi(\varepsilon, t)|^{2} d t\right) .
$$

We have: $\varphi(0, t)=D_{t} g(x)$. Let

$$
\begin{gathered}
\varphi_{1}(\varepsilon, t)=\frac{\partial}{\partial t} \Psi_{\varepsilon}^{t}\left(g_{t}(x)\right), \\
\varphi_{2}(\varepsilon, t)=d \Psi_{\varepsilon}^{t}\left(g_{t}(x)\right) \cdot \xi_{t}^{0}, \\
\varphi_{3}(\varepsilon, t)=\frac{1}{2} \sum_{i=1}^{m} \nabla\left(d \Psi_{\varepsilon}^{t}\right)\left(g_{t}(x)\right)\left(H_{i}(t), H_{i}(t)\right) .
\end{gathered}
$$

Since the torsion is free, we have

$$
\frac{D}{d \varepsilon} \varphi_{1}(\varepsilon, t)_{\left.\right|_{\varepsilon=0}}=\frac{D}{d \varepsilon} \frac{d}{d t} \Psi_{\varepsilon}^{t}\left(g_{t}(x)\right)_{\left.\right|_{\varepsilon=0}}=\left.\frac{D}{d t} \frac{d}{d \varepsilon}\right|_{\varepsilon=0} \Psi_{\varepsilon}^{t}\left(g_{t}(x)\right)=\alpha^{\prime}(t) w(x) .
$$

In order to compute the derivative of $\varphi_{2}$, consider a smooth curve $\beta(s) \in M$ such that $\beta(0)=g_{t}(x), \beta^{\prime}(0)=D_{t} g(x)$. Then

$$
d \Psi_{\varepsilon}^{t}\left(g_{t}(x)\right) \cdot \xi_{t}^{0}=\left.\frac{d}{d s}\right|_{s=0} \Psi_{\varepsilon}^{t}(\beta(s)) .
$$


Therefore

$$
\begin{aligned}
\left.\frac{D}{d \varepsilon}\right|_{\varepsilon=0} \varphi_{2}(\varepsilon, t) & =\left.\frac{D}{\left.d s\right|_{s=0}} \frac{d}{d \varepsilon}\right|_{\varepsilon=0} \Psi_{\varepsilon}^{t}(\beta(s))=\frac{D}{\left.d s\right|_{s=0}}[\alpha(t) w(\beta(s))] \\
& =\alpha(t)(\nabla w)\left(g_{t}(x)\right) \cdot D_{t} g(x) .
\end{aligned}
$$

For computing $\varphi_{3}$, we shall use another description given in [9] (p. 405). For the moment, consider a $C^{2}$ map $f: M \rightarrow M$. Let $x \in M$ and two tangent vectors $u, v \in T_{x} M$ be given. Let $x(t) \in M$ be a smooth curve such that $x(0)=x, x^{\prime}(0)=u$, and $Y_{t} \in T_{x_{t}} M$ such that $Y_{0}=v$. Define $Q(f)(x): T_{x} M \times T_{x} M \rightarrow T_{f(x)} M$ by

$$
Q(f)(x)(u, v)=\left.\frac{d}{d t}\right|_{t=0}\left[/ /_{t}^{-1}\left(d f\left(x_{t}\right) \cdot Y_{t}\right)\right]-d f(x) \cdot \nabla_{u} v .
$$

Then $\varphi_{3}$ can be expressed by

$$
\varphi_{3}(\varepsilon, t)=\frac{1}{2} \sum_{i=1}^{m} Q\left(\Psi_{\varepsilon}^{t}\left(g_{t}(x)\right)\right)\left(H_{i}(t), H_{i}(t)\right) .
$$

Let $\beta(s) \in M$ be a smooth curve such that $\beta(0)=g_{t}(x)$ and $\beta^{\prime}(0)=H_{i}(t)$ and $\left\{Y_{s} ; s \geq 0\right\}$ be a family of tangent vectors along $\{\beta(s) ; s \geq 0\}$ such that $Y_{0}=H_{i}(t)$. Set

$$
\gamma(\varepsilon, s)=\Psi_{\varepsilon}^{t}(\beta(s)) \quad \text { and } \quad X(\varepsilon, s)=d \Psi_{\varepsilon}^{t}(\beta(s)) \cdot Y_{s} .
$$

If $R$ denotes be the curvature tensor on $M$, the following commutation relation holds,

$$
\frac{D}{d \varepsilon} \frac{D}{d s} X(\varepsilon, s)=\frac{D}{d s} \frac{D}{d \varepsilon} X(\varepsilon, s)+R\left(\frac{\partial \gamma}{\partial \varepsilon}, \frac{\partial \gamma}{\partial s}\right) X(\varepsilon, s) .
$$

We have $X(0,0)=H_{i}(t), \frac{\partial \gamma}{\partial \varepsilon}(0,0)=\alpha(t) w(x), \frac{\partial \gamma}{\partial s}(0,0)=H_{i}(t)$; therefore

$$
\left[R\left(\frac{\partial \gamma}{\partial \varepsilon}, \frac{\partial \gamma}{\partial s}\right) X(\varepsilon, s)\right]_{\left.\right|_{\varepsilon=0, s=0}}=\alpha(t) R\left(w\left(g_{t}(x)\right), H_{i}(t)\right) H_{i}(t) .
$$

Now let $c(\tau) \in M$ be a smooth curve such that $c(0)=\beta(s), c^{\prime}(0)=Y_{s}$. We have

$$
\begin{aligned}
\left.\frac{D}{d \varepsilon}\right|_{\varepsilon=0} X(\varepsilon, s) & =\left[\frac{D}{d \tau} \frac{d}{d \varepsilon} \Psi_{\varepsilon}^{t}(c(\tau))\right](0,0) \\
& =\left.\alpha(t) \frac{D}{d \tau}\right|_{\tau=0} w(c(\tau))=\alpha(t)\left(\nabla_{Y_{s}} w\right)(\beta(s))
\end{aligned}
$$

and

$$
\left.\frac{D}{d s}\right|_{s=0}\left(\nabla_{Y_{s}} w\right)(\beta(s))=\left\langle\nabla_{H_{i}(t)} \nabla w, H_{i}(t)\right\rangle+\left\langle\nabla w, \nabla_{H_{i}(t)} H_{i}(t)\right\rangle
$$

Note that

$$
\left.\frac{D}{d \varepsilon}\right|_{\varepsilon=0} d \Psi_{\varepsilon}^{t}\left(g_{t}(x)\right) \cdot \nabla_{H_{i}(t)} H_{i}(t)=\alpha(t)\left\langle\nabla w, \nabla_{H_{i}(t)} H_{i}(t)\right\rangle .
$$

Using (2.21), we finally get

$$
\left.\frac{D}{d \varepsilon}\right|_{\varepsilon=0} \varphi_{3}(\varepsilon, t)=\frac{1}{2} \alpha(t) \sum_{i=1}^{m}\left[\left\langle\nabla_{H_{i}(t)} \nabla w, H_{i}(t)\right\rangle+R\left(w, H_{i}(t)\right) H_{i}(t)\right] .
$$


When $g_{t}$ is a $\nu$-Brownian semimartingale, the right hand side of above equality is equal to

$$
\nu \alpha(t)(\Delta w+\operatorname{Ric} w)\left(g_{t}(x)\right),
$$

which, due to (1.8), is equal to

$$
\nu \alpha(t)(-\hat{\square} w)\left(g_{t}(x)\right)
$$

In conclusion, $\frac{d}{d \varepsilon} S\left(\Psi_{\varepsilon}(g)\right)_{\left.\right|_{\varepsilon=0}}=0$ yields

$$
\mathbb{E}_{\mathbb{P} g} \int_{0}^{T}\left[\alpha^{\prime}(t) w\left(g_{t}\right) \cdot D_{t} g+\alpha(t)\left(\nabla_{D_{t} g} w\right)\left(g_{t}\right) \cdot D_{t} g-\nu \alpha(t) \hat{\square} w\left(g_{t}\right) \cdot D_{t} g\right] d t=0 .
$$

According to (2.18), the above equation is nothing but (2.19).

As a consequence of this result, we obtain

Theorem 2.11. Let $\left(u_{t}\right)_{t \in[0, T]}$ be a family of divergence free vector fields on $M$, which belong to the Sobolev space $\mathbb{D}_{1}^{2}$ and are such that

$$
\int_{M} \int_{0}^{T}\left(\left|u_{t}(x)\right|^{2}+\left|\nabla u_{t}(x)\right|^{2}\right) d t d x<+\infty
$$

then equations (2.7), (2.8) define an incompressible $\nu$-Brownian diffusion $\xi$ on $M$, which is a critical point of the action functional $S$ if and only if $u_{t}$ solves weakly the Navier-Stokes equation, that is,

$$
\int_{M} \int_{0}^{T}\left\langle u_{t}, \alpha^{\prime}(t) w+\alpha(t) \nabla w \cdot u_{t}-\nu \alpha(t) \hat{\square} w\right\rangle d t d x=0
$$

for all $\alpha \in C_{c}^{1}(] 0, T[)$ and all smooth vector fields $w \operatorname{such}$ that $\operatorname{div}(w)=0$.

Proof. First we notice that in Proposition 4.3 in [16], the condition $q>2$ insures the tightness of a family of probability measures; this condition can be relaxed to $q=2$ using Meyer-Zheng tightness results (see the proof of Theorem 2.13 below). Therefore by Theorem 6.4 in [16], equations (2.7) and (2.8) define a diffusion process $\xi$, which is, a fortiori, in $\mathcal{S}_{\nu}$. Therefore by the above computations (see (2.22)), $\xi$ is a critical point to $S$ if and only if

$$
\mathbb{E}_{\mathbb{P} g} \int_{0}^{T}\left[\alpha^{\prime}(t) w\left(\xi_{t}\right) \cdot u_{t}\left(\xi_{t}\right)+\alpha(t)\left(\nabla_{u_{t}\left(\xi_{t}\right)} w\right)\left(\xi_{t}\right) \cdot u_{t}\left(\xi_{t}\right)-\nu \alpha(t) \hat{\square} w\left(\xi_{t}\right) \cdot u_{t}\left(\xi_{t}\right)\right] d t=0,
$$

which yields the result.

Remark 2.12. It has been proved in [27] (see Theorem 4.6, p. 498) that for any $u_{0} \in$ $L^{2}(M, d x)$, there exists $\left\{u_{t}, t \in[0, T]\right\}$ solution to (2.24), satisfying Condition (2.23). Therefore equations (2.7), (2.8) define an incompressible $\nu$-Brownian diffusion $\xi$ on $M$, which is a critical point of the action functional $S$.

Note that in Theorem 3.2 of [4, a variational principle was established by using the first type of perturbations of identity, defined by (2.15); on the other hand the manifold $M$ was supposed there to be a symmetric space in order to insure the existence of semimartingales 
with the desired properties. A variational principe on a quite general Lie groups framework was derived in [3] (c.f. also [10]).

In [7, generalized flows with prescribed initial and final configuration were introduced. It is quite difficult to construct incompressible semimartingales with given prescriptions. In order to emphasize the contrast with the situation in [7, let's see the example of a Brownian bridge $g_{t}^{x, y}$ on $\mathbb{R}$ over $[0,1]$. It is known that for $t<1, g_{t}^{x, y}$ solves the following SDE

$$
d g_{t}^{x, y}=d w_{t}-\frac{g_{t}^{x, y}-y}{1-t} d t, \quad g_{0}^{x, y}=x .
$$

Then $g_{t}^{x, y} \rightarrow y$ as $t \rightarrow 1$ and we have

$$
\mathbb{E}\left(\int_{0}^{1}\left|D_{t} g^{x, y}\right|^{2} d t\right)=+\infty
$$

Let $\eta$ be a probability measure on $M \times M$ having $d x$ as two marginals; we shall say that the incompressible semimartingale $\left\{g_{t}\right\}$ has $\eta$ as final configuration if

$$
\mathbb{E}_{\mathbb{P}^{g}}\left(f\left(g_{0}, g_{T}\right)\right)=\int_{M \times M} f(x, y) d \eta(x, y), \quad f \in C(M \times M) .
$$

This means that the joint law of $\left(g_{0}, g_{T}\right)$ is $\eta$. If $g_{t}$ is as in Example 2.2, then

$$
\mathbb{E}_{\mathbb{P} g}\left(f\left(g_{0}, g_{T}\right)\right)=\int_{M \times M} f(x, y) p_{T}(x, y) d x d y,
$$

where $p_{t}(x, y)$ is the heat kernel associated to $\left(g_{t}\right)$. Conversely if $\left(\rho_{t}(x, y)\right)$ is solution to the following Fokker-Planck equation

$$
\frac{d}{d t} \rho_{t}(x, y)=\nu \Delta_{x} \rho_{t}(x, y)+\left\langle u_{t}(x), \nabla_{x} \rho_{t}(x, y)\right\rangle,
$$

with $\lim _{t \rightarrow 0} \rho_{t}=\delta_{x}$, for some $u \in L^{2}\left([0, T], \mathbb{D}_{1}^{2}(M)\right)$ with $\operatorname{div}\left(u_{t}\right)=0$, we can construct an incompressible $\nu$-Brownian semimartingale which has $\rho_{T}(x, y) d x d y$ as final configuration.

In any case, we have the following result:

Theorem 2.13. Let $\eta$ be a probability measure as above. If there exists an incompressible $\nu$-Brownian semimartingale $g$ on $M$ of finite energy $S(g)$ such that $\eta$ is its final configuration, then there exists one that minimizes the energy among all incompressible $\nu$-Brownian semimartingales having $\eta$ as final configuration.

Proof. Let $J: M \rightarrow \mathbb{R}^{N}$ be an isometric embedding; then $d J(x): T_{x} M \rightarrow \mathbb{R}^{N}$ is such that for each $x \in M$ and $v \in T_{x} M,|d J(x) \cdot v|_{\mathbb{R}^{N}}=|v|_{T_{x} M}$. Denote by $(d J(x))^{*}: \mathbb{R}^{N} \rightarrow T_{x} M$ the adjoint operator of $d J(x)$, that is,

$$
\left\langle(d J(x))^{*} a, v\right\rangle_{T_{x} M}=\langle d J(x) v, a\rangle_{\mathbb{R}^{N}}, \quad a \in \mathbb{R}^{N}, v \in T_{x} M .
$$

Let $\left\{\varepsilon_{1}, \ldots, \varepsilon_{N}\right\}$ be an orthonormal basis of $\mathbb{R}^{N}$ and set

$$
A_{i}(x)=(d J(x))^{*} \varepsilon_{i}, \quad i=1, \ldots, N .
$$

Then it is well-known that the vector fields $\left\{A_{1}, \ldots, A_{N}\right\}$ enjoy the following properties: 
(i) For any $v \in T_{x} M,|v|_{T_{x} M}^{2}=\sum_{i=1}^{N}\left\langle A_{i}(x), v\right\rangle_{T_{x} M}^{2}$.

(ii) $\sum_{i=1}^{N} \nabla_{A_{i}} A_{i}=0$.

Combining (i) and (ii) gives that $\Delta_{M} f=\sum_{i=1}^{N} \mathcal{L}_{A_{i}}^{2} f$ for any $f \in C^{2}(M)$. On the other hand, let $J(x)=\left(J_{1}(x), \ldots, J_{N}(x)\right)$; then

$$
\left\langle d J(x) v, \varepsilon_{i}\right\rangle=d J_{i}(x) \cdot v=\left\langle\nabla J_{i}(x), v\right\rangle_{T_{x} M}, \quad \text { for any } v \in T_{x} M .
$$

It follows that

$$
A_{i}=\nabla J_{i}, \quad i=1, \cdots, N
$$

Let $f \in C^{2}(M)$; then there exists $\bar{f} \in C^{2}\left(\mathbb{R}^{N}\right)$ such that $f(x)=\bar{f}(J(x))$. We have

$$
\begin{aligned}
\mathcal{L}_{A_{i}} f & =\sum_{j=1}^{N} \frac{\partial \bar{f}}{\partial x_{j}}(J(x))\left\langle\nabla J_{j}(x), A_{i}(x)\right\rangle \\
& =\sum_{j=1}^{N} \frac{\partial \bar{f}}{\partial x_{j}}(J(x))\left\langle A_{j}(x), A_{i}(x)\right\rangle .
\end{aligned}
$$

Therefore

$$
\begin{aligned}
\Delta_{M} f= & \sum_{i=1}^{N} \sum_{j, k=1}^{N} \frac{\partial^{2} \bar{f}}{\partial x_{j} \partial x_{k}}(J(x))\left\langle A_{j}, A_{i}\right\rangle\left\langle A_{k}, A_{i}\right\rangle \\
& +\sum_{i=1}^{N} \sum_{j=1}^{N} \frac{\partial \bar{f}}{\partial x_{j}}(J(x)) \mathcal{L}_{A_{i}}\left\langle A_{j}, A_{i}\right\rangle .
\end{aligned}
$$

Notice that

$$
\sum_{i=1}^{N} \mathcal{L}_{A_{i}}\left\langle A_{j}, A_{i}\right\rangle=\operatorname{div}\left(A_{j}\right)=\Delta_{M} J_{j}
$$

and according to property (i),

$$
\sum_{i=1}^{N}\left\langle A_{j}, A_{i}\right\rangle\left\langle A_{k}, A_{i}\right\rangle=\left\langle A_{j}, A_{k}\right\rangle
$$

Finally the Laplacian $\Delta_{M}$ on $M$ can be expressed by

$$
\Delta_{M} f=\sum_{j, k=1}^{N} \frac{\partial^{2} \bar{f}}{\partial x_{j} \partial x_{k}}(J(x))\left\langle A_{j}, A_{k}\right\rangle+\sum_{j=1}^{N} \frac{\partial \bar{f}}{\partial x_{j}}(J(x)) \Delta_{M} J_{j} .
$$

Having these preparations, we prove now the existence of a $g \in \mathcal{S}_{\nu}$ such that the minimum of action functinal $S$ is attained at $g$ in the class of those in $\mathcal{S}_{\nu}$ having $\eta$ as final configuration. Let

$$
K=\inf _{g \in S_{\nu}} S(g)
$$


There is a minimizing sequence $g^{n} \in \mathcal{S}_{\nu}$, that is, $\lim _{n \rightarrow+\infty} S\left(g^{n}\right)=K$. Consider the canonical decomposition:

$$
J\left(g_{t}^{n}\right)=J\left(g_{0}^{n}\right)+M_{t}^{n}+\int_{0}^{t} b^{n}(s) d s .
$$

Let $M_{t}^{n}=\left(M_{t}^{n, 1}, \cdots, M_{t}^{n, N}\right) ;$ then

$$
\left\langle M_{t}^{n, i}, M_{t}^{n, j}\right\rangle=2 \nu \int_{0}^{t}\left\langle\nabla J_{i}, \nabla J_{j}\right\rangle\left(g_{s}^{n}\right) d s
$$

By Itô formula, we have

$$
b^{n}(t)=d J\left(g_{t}^{n}\right) \cdot D_{t} g^{n}+\nu \Delta J\left(g_{t}^{n}\right)
$$

It follows that

$$
\mathbb{E}\left(\int_{0}^{T}\left|b^{n}(t)\right|^{2} d t\right) \leq 2 S\left(g^{n}\right)+2 T \nu\|\Delta J\|_{\infty}
$$

Therefore $\int_{0}^{T}\left|b^{n}(t)\right|^{2} d t$ is bounded in $L^{2}$. We can use Theorem 3 in [30] to conclude that the joint law $\hat{P}_{n}$ of

$$
\left(J\left(g^{n}\right), M_{\cdot}^{n}, B_{\cdot}^{n}, U_{\cdot}^{n}\right)
$$

in $C\left([0, T], \mathbb{R}^{N}\right) \times C\left([0, T], \mathbb{R}^{N}\right) \times C\left([0, T], \mathbb{R}^{N}\right) \times C\left([0, T], \mathbb{R}^{N \times N}\right)$ is a tight family, where

$$
B_{t}^{n}=\int_{0}^{t} b^{n}(s) d s, \quad U_{t}^{n}=\left(\left\langle M_{t}^{n, i}, M_{t}^{n, j}\right\rangle\right)_{1 \leq i, j \leq N} .
$$

Let $\hat{P}$ be a limit point; up to a subsequence, we suppose that $\hat{P}_{n}$ converges weakly to $\hat{P}$. Again by Theorem 3 in [30], under $\hat{P}$, the coordinate process

$$
\left(X_{t}, M_{t}, B_{t}, U_{t}\right)
$$

has the following properties:

(i) $M_{0}=B_{0}=0, U_{0}=0$,

(ii) $\left(M_{t}\right)$ is a local martingale such that $U_{t}=\left(\left\langle M_{t}^{i}, M_{t}^{j}\right\rangle\right)_{1 \leq i, j \leq N}$ and

(iii) $B_{t}=\int_{0}^{t} b(s) d s$ with $\int_{0}^{T}|b(s)|^{2} d s<+\infty$ almost surely.

Since $J(M)$ is closed in $\mathbb{R}^{N}$, we see that $X_{t} \in J(M)$. Let

$$
X_{t}=J\left(g_{t}\right) \text {. }
$$

For any $f \in C^{2}(M)$, by (2.30), we see that $f\left(g_{t}\right)$ is a real valued semimartingale. In other words, $\left\{g_{t} ; t \geq 0\right\}$ is a semimartingale on $M$. Let $f \in C(M)$, the map $f \circ J^{-1}: J(M) \rightarrow \mathbb{R}$ can be extended as a bounded continuous function on $\mathbb{R}^{N}$; therefore letting $n \rightarrow \infty$, we get

$$
\int_{M} f(x) d x=\mathbb{E}\left(f\left(g^{n}(t)\right)\right)=\mathbb{E}\left(f \circ J^{-1}\left(J\left(g_{t}^{n}\right)\right)\right) \rightarrow \mathbb{E}\left(f \circ J^{-1}\left(X_{t}\right)\right)=\mathbb{E}\left(f\left(g_{t}\right)\right) .
$$

In the same way, for $f \in C(M \times M)$, we have

$$
\int_{M \times M} f(x, y) d \eta(x, y)=\mathbb{E}\left(f\left(g^{n}(0), g^{n}(T)\right)\right)=\mathbb{E}\left(f\left(J^{-1} J\left(g^{n}(0)\right), J^{-1} J\left(g^{n}(T)\right)\right)\right)
$$


which goes to, as $n \rightarrow+\infty$,

$$
\mathbb{E}(f(g(0), g(T))) .
$$

So $g$ is incompressible and has $\eta$ as final configuration.

Besides, by (2.31), we have

$$
\left(\left\langle M_{t}^{i}, M_{t}^{j}\right\rangle\right)_{1 \leq i, j \leq N}=2 \nu \int_{0}^{t}\left\langle\nabla J_{i}, \nabla J_{j}\right\rangle\left(g_{s}\right) d s .
$$

Let $f \in C^{2}(M)$; denote by $M_{t}^{f}$ the martingale part of $f\left(g_{t}\right)$. Then by Itô formula,

$$
d M_{t}^{f}=\sum_{j=1}^{N} \frac{\partial \bar{f}}{\partial x_{j}}\left(X_{t}\right) d M_{t}^{j}
$$

Therefore for $f_{1}, f_{2} \in C^{2}(M)$, according to (2.33), we have

$$
\left\langle d M_{t}^{f_{1}}, d M_{t}^{f_{2}}\right\rangle=\sum_{j, k=1}^{N} \frac{\partial \bar{f}_{1}}{\partial x_{j}}\left(X_{t}\right) \frac{\partial \bar{f}_{2}}{\partial x_{k}}\left(X_{t}\right) 2 \nu\left\langle A_{j}, A_{k}\right\rangle_{g_{t}} d t .
$$

On the other hand, using relation (2.29) and property (i), we have

$$
\left\langle\nabla f_{1}, \nabla f_{2}\right\rangle=\sum_{\alpha=1}^{N} \mathcal{L}_{A_{\alpha}} f_{1} \mathcal{L}_{A_{\alpha}} f_{2}=\sum_{j, k=1}^{N} \frac{\partial \bar{f}_{1}}{\partial x_{j}} \frac{\partial \bar{f}_{2}}{\partial x_{k}}\left\langle A_{j}, A_{k}\right\rangle .
$$

Combinant above two equalities, we finally get

$$
\left\langle d M_{t}^{f_{1}}, d M_{t}^{f_{2}}\right\rangle=2 \nu\left\langle\nabla f_{1}, \nabla f_{2}\right\rangle_{g_{t}} d t .
$$

Since $X_{t}=J\left(g_{t}\right)$, we have

$$
d B_{t}=d J\left(g_{t}\right) \cdot D_{t} g d t+\frac{1}{2} \operatorname{Hess} J\left(g_{t}\right) d g_{t} \otimes d g_{t} .
$$

Relation (2.34) implies that $\frac{1}{2} \operatorname{Hess} J\left(g_{t}\right) d g_{t} \otimes d g_{t}=\nu \Delta_{M} J\left(g_{t}\right) d t$. Therefore we get

$$
B_{t}=\int_{0}^{t} d J\left(g_{s}\right) \cdot D_{s} g d s+\nu \int_{0}^{t} \Delta_{M} J\left(g_{s}\right) d s .
$$

In conclusion $\left\{g_{t} ; t \geq 0\right\}$ is a $\nu$-Brownian semimartingale on $M$ or $g \in \mathcal{S}_{\nu}$.

We want to see that $K=S(g)$. Firstly using the relation (2.32), for any $t \in[0, T]$,

$$
\int_{0}^{t} d J\left(g_{s}^{n}\right) \cdot D_{s} g^{n} d s=B_{t}^{n}-\nu \int_{0}^{t} \Delta J\left(g_{s}^{n}\right) d s .
$$

Let $\phi: C\left([0, T], \mathbb{R}^{N}\right) \rightarrow \mathbb{R}$ be a bounded continuous function, consider $\varphi: C\left([0, T], \mathbb{R}^{N}\right) \times$ $C\left([0, T], \mathbb{R}^{N}\right) \rightarrow \mathbb{R}$ defined by

$$
\varphi(B, g)=\phi\left(B .-\nu \int_{0}^{\cdot} \Delta J\left(g_{s}\right) d s\right) .
$$

Then $\varphi$ is a bounded continuous function on $C\left([0, T], \mathbb{R}^{N}\right) \times C\left([0, T], \mathbb{R}^{N}\right)$. It follows that $\int_{0} d J\left(g_{s}^{n}\right) \cdot D_{s} g^{n} d s$ converges in law to $\int_{0} d J\left(g_{s}\right) \cdot D_{s} g d s$. Let $\varepsilon>0$; for $n$ big enough, 


$$
\mathbb{E}\left(\int_{0}^{T}\left|d J\left(g_{s}^{n}\right) \cdot D_{s} g^{n}\right|^{2} d s\right) \leq K+\varepsilon
$$

Now by Theorem 10 in [24,

$$
\mathbb{E}\left(\int_{0}^{T}\left|d J\left(g_{s}\right) \cdot D_{s} g\right|^{2} d s\right) \leq K+\varepsilon
$$

or $\mathbb{E}\left(\int_{0}^{T}\left|D_{s} g\right|^{2} d s\right) \leq K+\varepsilon$. Letting $\varepsilon \rightarrow 0$ gives $S(g) \leq K$. So $S(g)=K$.

\section{Classical solutions and generalized paths}

In this section, $M$ will be a torus: $M=\mathbb{T}^{d}$. Let $g \in \mathcal{D}_{\nu}$ be the solution of the following $\mathrm{SDE}$ on $\mathbb{T}^{d}$

$$
d g_{t}=\sqrt{2 \nu} d w_{t}-u\left(T-t, g_{t}\right) d t, \quad g_{0} \in \mathbb{T}^{d}
$$

where $g_{0}$ is a random variable having $d x$ as law, $w_{t}$ is the standard Brownian motion on $\mathbb{R}^{d}$, and $\{u(t, x) ; t \in[0, T]\}$ is a family of $C^{2}$ vector fields on $\mathbb{T}^{d}$, identified to vector fields on $\mathbb{R}^{d}$ which are $2 \pi$-periodic with respect to each space component. Suppose that $u$ is a strong solution to the Navier-Stokes equation

$$
\frac{\partial}{\partial t} u(t, x)+\nabla u(t, x) \cdot u(t, x)-\nu \Delta u(t, x)=-\nabla p(T-t, x) .
$$

By Itô 's formula,

$$
\begin{aligned}
d u\left(T-t, g_{t}\right)= & -\left(\frac{\partial u}{\partial t}\right)\left(T-t, g_{t}\right)-\nabla u\left(T-t, g_{t}\right) \cdot u\left(T-t, g_{t}\right) \\
& +\nu \Delta u\left(T-t, g_{t}\right)+\sqrt{2 \nu} \nabla u\left(T-t, g_{t}\right) \cdot d w_{t} \\
= & \nabla p\left(t, g_{t}\right) d t+\sqrt{2 \nu} \nabla u\left(T-t, g_{t}\right) \cdot d w_{t}
\end{aligned}
$$

According to definition (2.2),$D_{t} g=-u\left(T-t, g_{t}\right)$ and

$$
D_{t} D_{t} g=-\nabla p\left(t, g_{t}\right)
$$

In what follows, we shall consider

$$
\mathcal{G}=\left\{g^{*} \in \mathcal{S}_{\nu} ; d g_{t}^{*}=\sqrt{2 \nu} d w_{t}+D_{t} g^{*} d t, g^{*}(0)=g(0), g^{*}(T)=g(T)\right\} .
$$

Note that semimartingales in $\mathcal{G}$ are defined on a same probability space.

Example 3.1. Let $\alpha$ be a real continuous function on $\mathbb{R}^{d}$ and set

$$
\beta(w, t)=\sin \left(\frac{\pi t}{T}\right) \int_{0}^{t} \alpha\left(w_{s}\right) d s, \quad c(w, t)=\frac{d}{d t} \beta(w, t) .
$$

Let $a \in \mathbb{R}^{d}$ be fixed. Consider $v(w, t)=c(w, t) a$; then $v$ is an adapted vector field on $\mathbb{T}^{d}$. Define

$$
g_{t}^{*}=g_{t}+\int_{0}^{t} v(w, s) d s .
$$

Then $g^{*} \in \mathcal{G}$. 
We have the following result

Theorem 3.2. Let $g \in \mathcal{D}_{\nu}$ be given in (3.1). Assume that the process $g$ is associated with the Navier-Stokes equation in the sense that

$$
D_{t} D_{t} g=-\nabla p\left(t, g_{t}\right)
$$

a.s. for a regular pression $p$ such that $\nabla^{2} p(t, x) \leq R \mathrm{Id}$, with $R T^{2} \leq \pi^{2}$. Then $g$ minimizes the energy $S$ in the class $\mathcal{G}$.

Proof. We define the following:

$$
B(g)=\frac{1}{2} \int_{0}^{T}\left|D_{t} g\right|^{2} d t-\int_{0}^{T} p(t, g(t)) d t
$$

Notice that the function $b(x, y)$ defined in [7] (p. 243) has no meaning in our setting (c.f. $(2.25)$ and $(2.26))$. Let $g^{*} \in \mathcal{G}$; we shall prove that

$$
\mathbb{E}(B(g)) \leq \mathbb{E}\left(B\left(g^{*}\right)\right)
$$

Consider the function

$$
\phi(t, x)=\frac{R}{2}|x|^{2}-p(t, x) .
$$

For each $t \geq 0$, the function $x \rightarrow \phi(t, x)$ is convex on $\mathbb{R}^{d}$ as $\nabla^{2} p(t, x) \leq R$ Id. By Itô formula

$$
d\left(D_{t} g \cdot g_{t}\right)=d\left(D_{t} g\right) \cdot g_{t}+\sqrt{2 \nu} D_{t} g \cdot d w_{t}+\left|D_{t} g\right|^{2} d t+d\left(D_{t} g\right) \cdot d g_{t} .
$$

Analogously,

$$
d\left(D_{t} g \cdot g_{t}^{*}\right)=d\left(D_{t} g\right) \cdot g_{t}^{*}+\sqrt{2 \nu} D_{t} g \cdot d w_{t}+D_{t} g \cdot D_{t} g^{*}+d\left(D_{t} g\right) \cdot d g_{t}^{*} .
$$

Remarking that $d\left(D_{t} g\right) \cdot d g_{t}=d\left(D_{t} g\right) \cdot d g_{t}^{*}$, and making the substraction of the above two equalities, we obtain

$$
d\left(D_{t} g \cdot\left(g_{t}^{*}-g_{t}\right)\right)=d\left(D_{t} g\right) \cdot\left(g_{t}^{*}-g_{t}\right)+\left(D_{t} g \cdot D_{t} g^{*}-\left|D_{t} g\right|^{2}\right) d t
$$

It follows that

$$
\begin{aligned}
& D_{T} g \cdot\left(g_{T}^{*}-g_{T}\right)-D_{0} g \cdot\left(g_{0}^{*}-g_{0}\right) \\
= & \int_{0}^{T} d\left(D_{t} g\right) \cdot\left(g_{t}^{*}-g_{t}\right)+\int_{0}^{T}\left(D_{t} g \cdot D_{t} g^{*}-\left|D_{t} g\right|^{2}\right) d t .
\end{aligned}
$$

Notice that $g_{0}^{*}=g_{0}, g_{T}^{*}=g_{T}$, and using (3.1), we have

$$
\begin{aligned}
& \int_{0}^{T}\left(-D_{t} g \cdot D_{t} g^{*}+\left|D_{t} g\right|^{2}\right) d t=\int_{0}^{T} d\left(D_{t} g\right) \cdot\left(g_{t}-g_{t}^{*}\right) \\
& =\int_{0}^{T}\left(g_{t}^{*}-g_{t}\right) \cdot\left(-\sqrt{2 \nu} \nabla u\left(T-t, g_{t}\right) d w_{t}-\nabla p\left(t, g_{t}\right) d t\right) .
\end{aligned}
$$

Using the convexity, of $\phi$, we have

$$
\phi\left(t, g_{t}^{*}\right)-\phi\left(t, g_{t}\right) \geq\left(R g_{t}-\nabla p\left(t, g_{t}\right)\right) \cdot\left(g_{t}^{*}-g_{t}\right) .
$$


From (3.7) and (3.8), we get

$$
\begin{aligned}
& \int_{0}^{T}\left(-D_{t} g \cdot D_{t} g^{*}+\left|D_{t} g\right|^{2}+R g_{t} \cdot\left(g_{t}^{*}-g_{t}\right)\right) d t \\
\leq & -\sqrt{2 \nu} \int_{0}^{T}\left(g_{t}^{*}-g_{t}\right) \cdot \nabla u\left(T-t, g_{t}\right) d w_{t}+\int_{0}^{T}\left(\phi\left(t, g_{t}^{*}\right)-\phi\left(t, g_{t}\right)\right) d t .
\end{aligned}
$$

We have $g_{t}^{*}-g_{t}=\int_{0}^{t}\left(D_{s} g^{*}-D_{s} g\right) d s$. Since $g_{0}^{*}-g_{0}=g_{T}^{*}-g_{T}=0$, by Poincaré 's inequaliy on the circle to get

$$
\int_{0}^{T}\left|g_{t}^{*}-g_{t}\right|^{2} d t \leq\left(\frac{T}{\pi}\right)^{2} \int_{0}^{T}\left|D_{t} g^{*}-D_{t} g\right|^{2} d t .
$$

Since $\left(\frac{T}{\pi}\right)^{2} \leq \frac{1}{R}$, we have

$$
\frac{R}{2} \int_{0}^{T}\left|g_{t}^{*}-g_{t}\right|^{2} d t \leq \frac{1}{2} \int_{0}^{T}\left|D_{t} g^{*}-D_{t} g\right|^{2} d t .
$$

Remark that the inequality, for $x, y, a, b \in \mathbb{R}$

$$
x^{2}-x y-R b^{2}+R a b \geq \frac{1}{2} x^{2}-\frac{1}{2} y^{2}-\frac{R}{2} b^{2}+\frac{R}{2} a^{2}
$$

holds if and only if

Therefore by (3.10), we have

$$
\frac{1}{2}(x-y)^{2} \geq \frac{R}{2}(b-a)^{2} .
$$

$$
\begin{aligned}
& \int_{0}^{T}\left(\left|D_{t} g\right|^{2}-D_{t} g \cdot D_{t} g^{*}-R\left|g_{t}\right|^{2}+R g_{t} \cdot g_{t}^{*}\right) d t \\
& \geq \int_{0}^{T}\left(\frac{1}{2}\left|D_{t} g\right|^{2}-\frac{1}{2}\left|D_{t} g^{*}\right|^{2}-\frac{R}{2}\left|g_{t}\right|^{2}+\frac{R}{2}\left|g_{t}^{*}\right|^{2}\right) d t .
\end{aligned}
$$

Combining (3.9) and (3.11), we get

$$
\begin{aligned}
& \int_{0}^{T}\left(\frac{1}{2}\left|D_{t} g\right|^{2}-\frac{1}{2}\left|D_{t} g^{*}\right|^{2}-\frac{R}{2}\left|g_{t}\right|^{2}+\frac{R}{2}\left|g_{t}^{*}\right|^{2}\right) d t \\
\leq & -\sqrt{2 \nu} \int_{0}^{T}\left(g_{t}^{*}-g_{t}\right) \cdot \nabla u\left(T-t, g_{t}\right) d w_{t}+\int_{0}^{T}\left(\phi\left(t, g_{t}^{*}\right)-\phi\left(t, g_{t}\right)\right) d t,
\end{aligned}
$$

from which we deduce

$$
\begin{aligned}
& \int_{0}^{T}\left(\frac{1}{2}\left|D_{t} g\right|^{2}-\frac{R}{2}\left|g_{t}\right|^{2}+\phi\left(t, g_{t}\right)\right) d t \\
& \leq-\sqrt{2 \nu} \int_{0}^{T}\left(g_{t}^{*}-g_{t}\right) \cdot \nabla u\left(T-t, g_{t}\right) d w_{t}+\int_{0}^{T}\left(\frac{1}{2}\left|D_{t} g^{*}\right|^{2}-\frac{R}{2}\left|g_{t}^{*}\right|^{2}+\phi\left(t, g_{t}^{*}\right)\right) d t
\end{aligned}
$$

or

$$
\begin{gathered}
\int_{0}^{T}\left(\frac{1}{2}\left|D_{t} g\right|^{2}-p\left(t, g_{t}\right)\right) d t \\
\leq-\sqrt{2 \nu} \int_{0}^{T}\left(g_{t}^{*}-g_{t}\right) \cdot \nabla u\left(T-t, g_{t}\right) d w_{t}+\int_{0}^{T}\left(\frac{1}{2}\left|D_{t} g^{*}\right|^{2}-p\left(t, g_{t}^{*}\right)\right) d t .
\end{gathered}
$$


Using definition (3.5),

$$
B(g) \leq-\sqrt{2 \nu} \int_{0}^{T}\left(g_{t}^{*}-g_{t}\right) \cdot \nabla u\left(T-t, g_{t}\right) d w_{t}+B\left(g^{*}\right) .
$$

Taking the expectation of this inequality, we obtain (3.6). Notice that $\int_{0}^{T} \mathbb{E}\left(p\left(t, g_{t}\right)\right) d t=$ $\int_{0}^{T} \mathbb{E}\left(p\left(t, g_{t}^{*}\right)\right) d t$; then (3.6) yields $\mathbb{E}(S(g)) \leq \mathbb{E}\left(S\left(g^{*}\right)\right)$.

The following result provides a perturbation in a natural way and illustrates Theorem 3.2 .

Proposition 3.3. Let $v(w, t)$ be the vector field constructed in Example 3.1. Consider the following perturbation of $g_{t}$ given by (3.1):

$$
d g_{t}^{\varepsilon}=\sqrt{2 \nu} d w_{t}-u\left(T-t, g_{t}\right) d t+\varepsilon v(w, t) d t, \quad g_{0}^{\varepsilon}=x .
$$

Then we have

$$
\frac{d}{d \varepsilon} S\left(g^{\varepsilon}\right)_{\left.\right|_{\varepsilon=0}}=0
$$

Proof. We see that $\left\{g^{\varepsilon} ; \varepsilon \geq 0\right\} \subset \mathcal{G}$. We have

$$
S\left(g^{\varepsilon}\right)=\frac{1}{2} \mathbb{E}\left(\int_{0}^{T}\left|u\left(T-t, g_{t}\right)-\varepsilon v(w, t)\right|^{2} d t\right) .
$$

Therefore

$$
\frac{d}{d \varepsilon} S\left(g^{\varepsilon}\right)_{\left.\right|_{\varepsilon=0}}=-\mathbb{E}\left(\int_{0}^{T}\left\langle u\left(T-t, g_{t}\right), v(w, t)\right\rangle d t\right)
$$

Let $V_{t}=\int_{0}^{t} v_{s} d s$. By construction of $v, V_{T}=0$. Now by integration by parts,

$$
-\int_{0}^{T}\left\langle u\left(T-t, g_{t}\right), \dot{V}(w, t)\right\rangle d t=\int_{0}^{T}\left\langle d\left(u\left(T-t, g_{t}\right)\right), V(w, t)\right\rangle d t
$$

which is equal to, using (3.2), $\int_{0}^{T}\left\langle\nabla p\left(t, g_{t}\right), V(w, t)\right\rangle d t$. Therefore

$$
\begin{aligned}
\frac{d}{d \varepsilon} S\left(g^{\varepsilon}\right)_{\left.\right|_{\varepsilon=0}}= & \int_{0}^{T} \mathbb{E}\left(\int_{\mathbb{T}^{d}}\left\langle\nabla p\left(t, g_{t}(x)\right), \beta(w, t) a\right\rangle d x\right) d t \\
& =\int_{0}^{T} \mathbb{E}(\beta(w, t))\left(\int_{\mathbb{T}^{d}}\langle\nabla p(t, x), a\rangle d x\right) d t=0 .
\end{aligned}
$$

\section{References}

[1] L. Ambrosio, A. Figalli, Geodedics in the space of measure-preserving maps and plans, Arch. Rational Mech. Anal., 194 (2009), 421-469.

[2] A. Antoniouk, M. Arnaudon, A.B. Cruzeiro, Generalized stochastic flows and applications to incompressible viscous fluids, Bull. Sci. Math., vol 138, issue 4 (2014), p. $565-584$.

[3] M. Arnaudon, X. Chen and A.B. Cruzeiro, Stochastic Euler-Poincaré reduction, J. Math. Physics, 55 (2014), 081507. 
[4] M. Arnaudon, A.B. Cruzeiro, Lagrangian Navier-Stokes diffusions on manifolds: variational principle and stability, Bull. Sci. Math., 136 (8) (2012), p. 857-881.

[5] M. Arnaudon, A.B. Cruzeiro, Stochastic Lagrangian flows on some compact manifolds, Stochastics, 84 (2-3) (2012), p. 367-381.

[6] V. I. Arnold, Sur la géométrie différentielle des groupes de Lie de dimension infinie et ses applications à l' hydrodynamique des fluides parfaits, Ann. Inst. Fourier, 16 (1966), 316-361.

[7] Y. Brenier, The least action principle and the related concept of generalized flows for incompressible perfect fluids. J. AMS, 2 (1989), 225-255.

[8] Y. Brenier, Minimal geodesics on groups of volume-preserving maps and generalized solutions of the Euler equations, Comm. Pure and Appl. Maths, 52 (1999), 411-452.

[9] J.M. Bismut, Mécanique aléatoire, Lect. Notes in Maths, 866, 1981

[10] X. Chen, A.B. Cruzeiro, T. Ratiu, Constrained and stochastic variational principles for dissipative equations with advected quantities, arXiv:1506.05024

[11] P.Constantin, G. Iyer, A stochastic Lagrangian representation of the threedimensional incompressible Navier-Stokes equations, Comm. Pure Appl. Math., 61 (2008), 330-345.

[12] F. Cipriano and A.B. Cruzeiro, Navier-Stokes equations and diffusions on the group of homeomorphisms of the torus, Comm. Math. Phys. 275 (2007), 255-269.

[13] R. DiPerna, A. Majda, Oscillations and concentrations in weak solutions of incompressible fluid equations, Comm. Math. Phys., 108 (1987), 667-689.

[14] D.G. Ebin, J.E. Marsden, Groups of diffeomorphisms and the motion of an incompressible fluid, Ann. of Math. 92 (1970), 102-163.

[15] G. L. Eyink, Stochastic least-action principle for the incompressible Navier-Stokes equation, Physica D, 239 (2010), 1236-1240.

[16] S. Fang, H.Li, D. Luo, Heat semi-group and generalized flows on complete Riemannian manifolds. Bull. Sci. Math., 135 (2011), 565-600.

[17] S. Fang, D. Luo, A. Thalmaier, Stochastic differential equations with coefficients in Sobolev spaces. J. Funct. Analysis, 259 (2010), 1129-1168.

[18] W.H. Fleming, H.M. Soner, Controlled Markov processes and viscosity solutions, Stoch. Modelling and Applied Prob. 25, Springer, Berlin, 2006. (2nd ed.)

[19] H. Follmer, Random fields and diffusion processes. École d'été de Probabilités de Saint-Flour, XV-XVII-1982-87, 143-303, Lecture Notes in Math., 1362, Springer, Berlin, 1988.

[20] G. Iyer, A variational principle for the Navier-Stokes equations, preprint, 2008.

[21] H. Kunita, Stochastic differential equations and stochastic flows of diffeomorphisms. École d'été de Probabilités de Saint-Flour, XII-1982, 143-303, Lecture Notes in Math., 1097, Springer, Berlin, 1984. 
[22] H. Kunita, Stochastic Flows and Stochastic Differentail Equations. Cambridge University Press, 1990.

[23] C. Léonard, A survey of the Schrödinger problem and some of its connections with optimal transport, Discrete Contin. Dyn. Syst., 34 (2014), 1533-1574.

[24] P.A. Meyer, W.A. Zheng, Tightness criteria for laws of semimartingales, Annales de l'I.H.P., section B, tome 20, n. 4 (1984), 353-372.

[25] M. Mitrea, M. Taylor, Navier-Stokes equations on Lipschitz domains in Riemannian manifolds, Math. Ann., 321(2001), 955-987.

[26] V. Pierfelice, The incompressible Navier-Stokes equations on non-compact manifolds, arXive: 1406.1644v1.

[27] M. Taylor, Partial Differential Equations III: Nonlinear Equations, Nonlinear equations, Vol. 117, Applied Mathematical Sciences, Springer New York second edition (2011).

[28] K. Yasue, A variational principle for the Navier-Stokes equation, J. Funct. Anal., 51 (2), (1983) 133-141.

[29] J.C. Zambrini, Variational processes and stochastic versions of Mechanics. J. Math. Phys 27 (9), (1986), 2307

[30] W. A. Zheng, Tightness results for laws of diffusion processes. Ann. de l' I.H.P., section B 21 (2) (1985), 103-124. 